\title{
Polystyrene Modified by Grafting
}

\author{
Avani Maria C. Rocha ${ }^{a}$, Laura Hecker de Carvalho ${ }^{b}$, \\ and Antonio Gouveia de Souza*c \\ ${ }^{a}$ Departamento de Físico-Química, I.Q, Universidade Federal da Bahia, \\ 40170-280 Salvador - BA, Brazil \\ ${ }^{b}$ Departamento de Engenharia de Materiais, DEMa/CCT, Universidade Federal da \\ Paraíba, 58109-970 Campina Grande - PB, Brazil \\ ${ }^{c}$ Departamento de Química, DQ/CCEN, Universidade Federal da Paraíba, \\ 58059-900 João Pessoa - PB, Brazil
}

Received: March 20, 1995

\begin{abstract}
Diversos tipos de poliestireno modificados foram obtidos através da polimerização por enxertia (graft polymerization) de estireno na presença dos seguintes modificadores: copolímero de etileno-acetato de vinila (EVA); polietileno de baixa densidade (PE) e polibutadieno (PB). Os modificadores foram utilizados em separado e combinados. Em todos os casos, manteve-se a concentração de modificador fixa em $8 \%$ em peso e foram avaliadas as propriedades dos produtos obtidos em função da sua composição. A técnica de polimerização empregada foi a de massa/suspensão. Os melhores resultados foram obtidos para os sistemas PS/modificadores mistos. Os resultados obtidos para estes sistemas comprovam que a copolimerização por enxertia gera produtos com melhores propriedades de impacto do que as obtidas, a partir de misturas mecânicas dos componentes individuais, em sistemas semelhantes.
\end{abstract}

Several grades of modified polystyrene were obtained through the graft polymerization of styrene in the presence of modifiers such as ethylene-vinyl acetate (EVA), low density polyethylene (PE), and polybutadiene (PB). These modifiers were used by themselves and in combination. In all cases, the modifier concentration was kept constant at $8 \% \mathrm{w} / \mathrm{w}$, and the product properties were determined as a function of composition. The polymerization technique employed was that of mass/suspension. The best results were obtained for the PS/mixed modifiers systems. The results confirm that graft copolymers present better impact properties than those of mechanical blends of similar composition.

Keywords: polystyrene, grafting, polybutadiene, ethylene-vinyl acetate, polyethylene

\section{Introduction}

Polystyrene (PS) is a non-crystalline thermoplastic widely used for many applications, and which, despite its good mechanical properties, high gloss and easy processing, exhibits low stress-cracking resistance and is considered too brittle for many applications ${ }^{1-2}$. In order to improve polystyrene toughness, attempts were made to modify its impact characteristics. This is usually done by incorpora- ting polybutadiene (PB), which is elastomeric, as dispersed particles in the brittle PS matrix. Rubber-modified PS is known as high impact polystyrene (HIPS), and can be made by two methods: a) mechanical blending or b) graft copolymerization of PS in the presence of the elastomer.

Polyolefins, in general, have good stress-cracking resistance, easy processing, and are tougher than PS. Therefore, it was thought that mechanical blending of PS with polyole- 
fins would be able to resolve the drawbacks ${ }^{3}$ presented by PS, i.e., low stress-cracking resistance and brittleness. In doing so, however, researchers found yet another problem which deals with the fact that most of these blends are not miscible ${ }^{4}$ or even compatible and therefore the final products tend to have poor mechanical properties.

In order for a given blend to present good properties, the dispersed phase must be homogeneously dispersed, stable, and without a tendency to agglomerate. In general, either in composite or blend development, a good interface is the key to ensuring good properties as stress transfer and crack propagation occur in that region ${ }^{5}$. One of the means to promote a strong interface is to add compatibilizing agents to the blends or the composites. These agents are usually amphoteric or amphiphilic molecules, i.e. molecules composed of moieties with affinities for both components of the system being analyzed. Among the compatibilizing agents employed in the development of PS/PE blends, the most popular are graft copolymers of PS/PB or hydrogenated styrene/butadiene block copolymers ${ }^{6-10}$.

A great deal of effort has been made to develop compatible blends of immiscible PS/polyolefin systems displaying good mechanical properties. In general, this is done through the addition of compatibilizers by mechanical blending. Some work has been done on the improvement of mechanical properties of HIPS/EVA blends through the use of styrene/butadiene/styrene (SBS) tri-block copolymers. Except for a patent ${ }^{11}$, no reports were found on the development of PS/PE or PS/EVA blends by graft copolymerization.

In this work, PS/polyolefin blends obtained by graft copolymerization of PS in the presence of several impact modifiers, used by themselves or in combination, were mechanically and thermo-mechanically characterized. The matrix molecular weight was monitored by size exclusion chromatography (SEC), and the particle size (volume average particle diameter $-\bar{D}_{\mathrm{v}}$ and number average particle diameter $-\bar{D}_{\mathrm{n}}$ ) of the elastomeric inclusion, was determined with a Coulter counter analyzer.

\section{Experimental}

Impact-modifed PS was obtained in a two-stage (mass/ suspension) styrene graft copolymerization process with a typical HIPS procedure and formulation. The reaction composition employed is shown in Table 1 . In the first stage of the polymerization process employed, styrene was pre-polymerized (up to $30 \%$ conversion, mass polymerization process) in the presence of modifiers, initiator (trigonox), chain transfer agent (mercaptan), stabilizer (irganox), and mineral oil. The reaction took place in a glass reactor under nitrogen atmosphere and constant stirring (200 rpm) as the temperature was raised and then kept constant at 95 ${ }^{\circ} \mathrm{C}$. In the second stage (suspension polymerization), which took place immediately after mass polymerization, water
Table 1. Formulation of the styrene-grafted impact modifiers.

\begin{tabular}{lc}
\hline Reagents & \% weight \\
\hline Styrene & 90.00 \\
Modifier & 8.00 \\
Peroxicyclohexane & 0.10 \\
Mercaptan & 0.05 \\
Antioxidant & 0.05 \\
Mineral oil & 1.50 \\
Benzoyl peroxide & 0.20 \\
T-butyl perbenzoate & 0.10 \\
\hline
\end{tabular}

and poly(vinyl alcohol) were added to the reaction mixture, the stirring speed was increased to $450 \mathrm{rpm}$, the temperature was kept at $91{ }^{\circ} \mathrm{C}$, the remaining initiators (BPO and perbenzoate) were added, and the reaction was allowed to proceed up to $98 \%$ conversion. The reaction contents were then transferred to a stainless steel (PARR) reactor in order to complete the polymerization, i.e., to consume the remaining initiator and to polymerize the residual styrene monomer. This polymerization stage is called the "curing stage", and took place by heating the reaction mixture for $1 \mathrm{~h}$ at 115 ${ }^{\circ} \mathrm{C}$, followed by $1 \mathrm{~h}$ at $135^{\circ} \mathrm{C}$. The resulting polymer was washed, filtered, oven-dried at $60{ }^{\circ} \mathrm{C}$, and stored for characterization. The procedure employed in this work ensures good mixing, proper particle size, and dispersion of the rubber phase. The product obtained consists of a continuous PS matrix in which small domains of the modifier are embedded. It is believed that grafting occurs by termination (coupling) of growing PS macro-radicals with modifier macro-radicals formed by chain transfer reactions ${ }^{1,12}$, and that the graft copolymer concentrates at the interface ${ }^{12}$. In all reactions under investigation, modifier content was kept at $8 \%$, as this is the typical value of the PB content for HIPS packaging applications.

Seven products with $8 \%$ total modifier content were studied. The modifiers employed were: a) polybutadiene (PB), medium-cis content, provided by COPERBO; b) styrene-butadiene-styrene (SBS) block copolymer, coded TR-2040, provided by COPERFLEX; c) low-density polyethylene (PE), coded F-0321, provided by POLITENO and d) ethylene-vinyl acetate copolymer (EVA) with 19\% vinyl acetate content, coded PE-3019, provided by POLITENO. The modifier contents and combinations investigated in this work were: $8 \% \mathrm{~PB} ; 8 \% \mathrm{PE} ; 8 \%$ EVA; $4 \%$ $\mathrm{PB}+4 \% \mathrm{PE} ; 4 \% \mathrm{~PB}+4 \% \mathrm{EVA} ; 4 \% \mathrm{~PB}+2 \% \mathrm{PE}+2 \%$ $\mathrm{SBS}$, and $4 \% \mathrm{~PB}+2 \% \mathrm{EVA}+2 \% \mathrm{SBS}$.

The products were characterized by:

a) Izod impact strength according to ASTM D 256-81, which was performed on notched injected test specimens using a BLI Impact Tester from SATEC Systems; 
b) Chemical stress-cracking, following a procedure adopted for testing packaging material, which was performed at $24{ }^{\circ} \mathrm{C}$ under $1871 \mathrm{psi}$ on compressed rectangular samples having nominal dimensions of $150 \times 13 \times 3 \mathrm{~mm}$, immersed in an oil bath composed of a 1:1 mixture of soy bean oil and oleic acid;

c) Particle size analysis performed on a Coulter counter analyzer, model TA I;

d) Melt flow index of all products determined on a TINIUS OLSEN apparatus operating according to ASTM D-1231, G condition;

e) Tensile testing performed according to ASTM D-638/80, on type IV injected samples, on an INSTRON-1131 testing machine operating at $50 \mathrm{~mm} / \mathrm{min}$ at $24{ }^{\circ} \mathrm{C}$. The parameters determined were tensile strength at yield and at break, elongation at break, and elastic modulus;

f) Molecular weight and molecular weight distribution of the PS matrix determined by size exclusion cromatography (SEC), according to ASTM D-3593, on a VARIAN LC system apparatus operating with TSK columns and a $\mathrm{UV} / \mathrm{V}$ is detector at $257 \mathrm{~nm}$, using THF as a solvent, and a pumping rate of $1 \mathrm{~mL} / \mathrm{min}$ at $25^{\circ} \mathrm{C}$; and

g) Dynamic mechanical analysis (DMA) performed on a DUPONT model DMA 982, dynamic mechanical analyzer operating at a heating rate of $5{ }^{\circ} \mathrm{C} / \mathrm{min}$ from $-130{ }^{\circ} \mathrm{C}$ to $100{ }^{\circ} \mathrm{C}$, at a $0.2 \mathrm{~mm}$ oscillating amplitude and a $30 \mathrm{~Hz}$ frequency.

\section{Results and Discussion}

Table 2 summarizes the results obtained for all systems under investigation. With respect to the values of molecular weight and molecular weight distribution of the polystyrene matrices, it is evident that, within experimental error, the nature of the modifier did not cause relevant changes in these parameters ${ }^{14-15}$. This is to be expected, since all reactions were carried out under the same experimental conditions of temperature, modifier content and initiator concentrations, and the only parameter varied was the nature of the modifier. Graft copolymers are obtained by the polymerization of a given monomer in the presence of a polymeric substrate, and consist of branched structures with a backbone of one monomer to which side chains of another monomer are attached. It is assumed that chain transfer is responsible for branching, the exact mechanism still being subject to discussion, but generally speaking, it is believed that free-radicals are created onto the polymeric substrate by hydrogen abstraction, and that grafting occurs by termination (combination) of this macro-radical with a growing polymer chain ${ }^{1-2}$, rather than by reinitiation with the macro-radical. A schematic representation of this mechanism is given in Scheme 1.

According to the scheme above, a growing polymer chain $(\mathrm{Mn} \bullet)$ abstracts a hydrogen from another polymer chain by a chain transfer reaction, generating a macro-radical. A graft copolymer is produced either by ma-

Table 2. Structure and Properties of the Modified Polystyrenes.

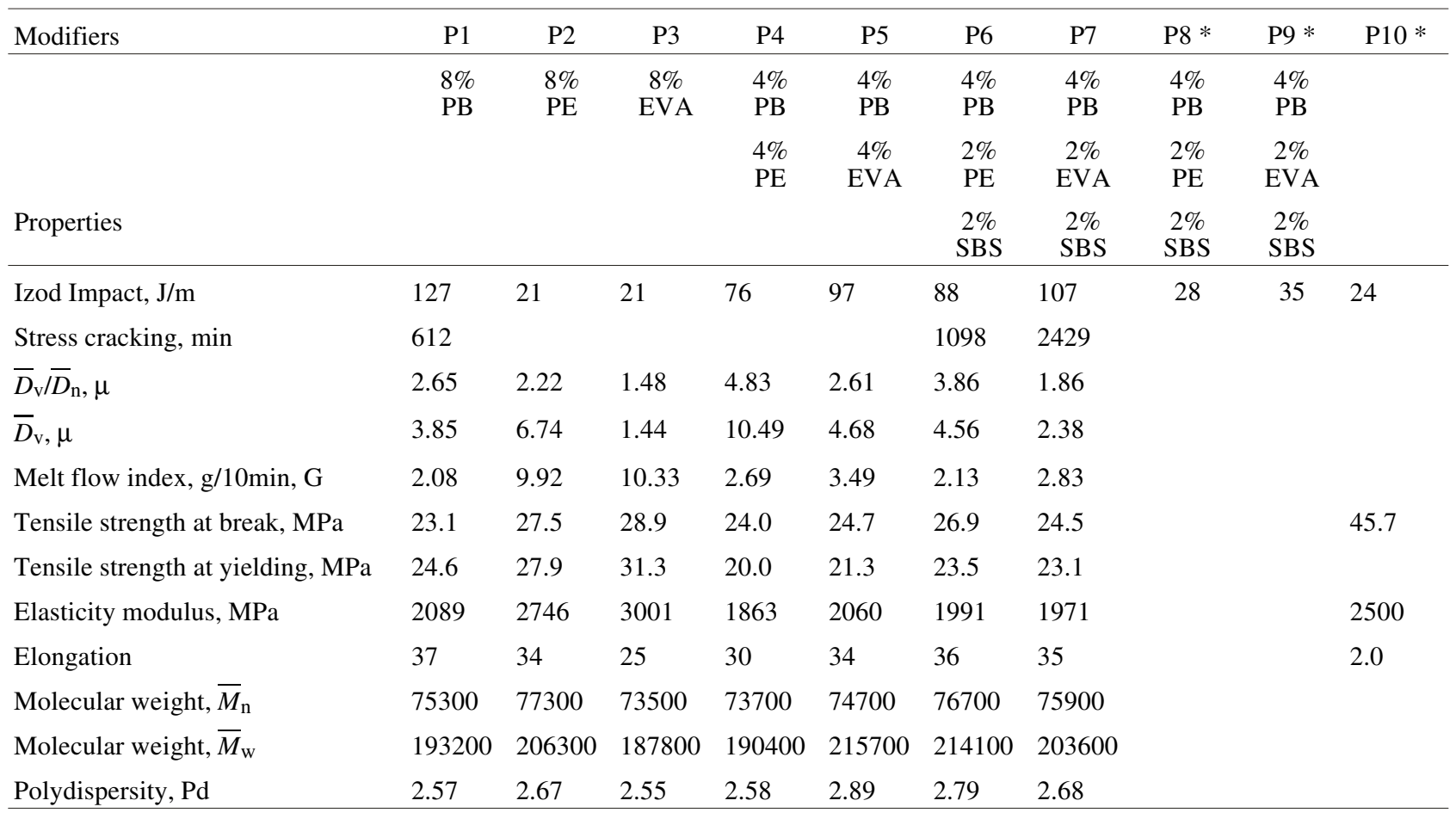

* P8 and P9 refer to mechanical blends of composition similar to P6 and P7, respectively, and P10 to polystyrene. 


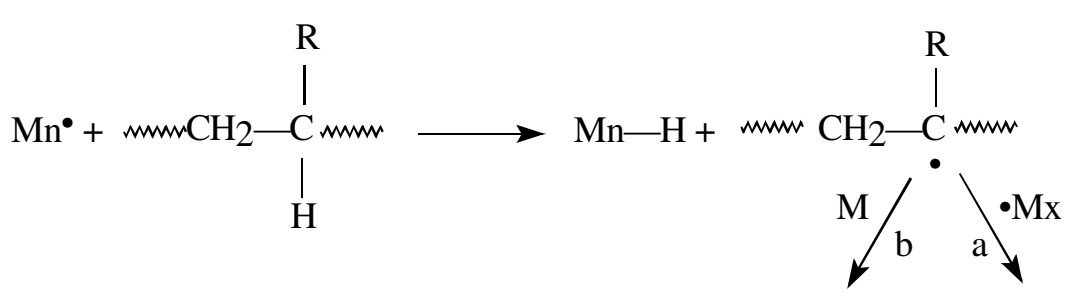

Scheme 1.

cro-radical reinitiation (b) or by coupling (termination) with another growing polymer chain (a).

Polymer molecular weight is strongly dependent on polymerization temperature and initiator concentration, and except for a chain transfer to the polymer, is reduced by all other chain transfer reactions. In general, a chain transfer to the polymer is not extensive under mild reaction conditions such as the ones employed here, but should not be neglected for polymerizations carried out to complete or high conversion ${ }^{2}$. Considering that all experiments were carried out under the same experimental conditions, it was expected that if changes in molecular weight as a function of modifier nature were to be detected, these could be assigned to chain transfer characteristics of the modifiers employed. Our results seem to indicate that the extent of chain transfer reactions was essentially the same for all systems investigated, as no significant variation in polystyrene molecular weight was observed.

As expected, the melt flow index (MFI) of the products (Table 2) showed modifier addition strongly affecting this property. This is due to the fact that PE and EVA have much lower viscosities, and therefore higher flow properties, than PB or SBS. As expected, the addition of SBS to the mixed systems increased the systems overall viscosity, lowering their MFI.

Table 2 also presents the values for the average particle size and particle size distribution obtained for all systems investigated. It is evident from the volume average particle diameter $\left(\bar{D}_{\mathrm{v}}\right)$ values, that the addition of $\mathrm{PE}$ as a modifier produced the largest particles, while the addition of EVA generated smaller particles. The particle size distribution also tended to narrow whenever EVA was added to the system. The values of particle size and particle size dispersity for the mixed modifier systems (PB/PE and PB/EVA) were larger than what would be expected by the averaging of individual values, indicating a more heterogeneous system. The addition of SBS as a compatibilizer seemed to be effective as, in this case, both particle size and particle size dispersity noticeably decreased. The particle size and particle size distribution of the modifier have a strong influence on the final properties ${ }^{13}$ of the blends investigated. Particles which are too large or too heterogeneous in size tend to concentrate stresses, lowering the impact resistance and tensile strength of the products. On the other hand, particles which are too small, although not strongly affecting the products' tensile strength, lower the impact strength of the product, as they are unable to effectively dissipate tension during craze propagation. In general, the best impact properties are obtained with particle sizes in the range of 1-6 $\mu \mathrm{m}$, the optimum particle size being dependent on each particular system ${ }^{16-18}$ under study.

The tensile properties of all systems under study are presented in Table 2. As expected, modifier incorporation lowered the tensile strength and modulus and increased the elongation at the breaking of the products investigated. This is due to the fact that PS is a hard, brittle plastic, while the modifiers employed (PB, PE and EVA) are softer and tougher than PS. The best tensile properties were obtained for PS/EVA and PS/PE systems, as these modifiers (EVA and $\mathrm{PE}$ ) have higher tensile strength and modulus than $\mathrm{PB}$, i.e., are relatively strong and rigid compared to $\mathrm{PB}$. The combination of highest tensile strength and modulus was obtained for the system PS/EVA, which is attributed to the properties of EVA (higher tensile strength and modulus, and lower elongation at break), as well as to the smaller particle diameter and interparticle distance presented by this system $^{12}$. In general, the mechanical properties of the mixed systems analyzed exhibited an intermediate behavior between that of PS/PB and PS/PE or PS/EVA. Among the compatibilized systems, i.e. those with SBS, a slightly better tensile strength was obtained for the quaternary PS/PB/PE/SBS system.

The impact strength of all systems analyzed (Table 2) shows that the best properties were obtained for the PS/PB system, and that the PS/PE and PS/EVA systems showed poor impact properties. Partial replacement of $\mathrm{PB}$ with $\mathrm{PE}$ 
or EVA did lower the impact strength of the products with respect to that of $\mathrm{PS} / \mathrm{PB}$, but this change was not enough to prevent this product from being used as a toughened PS. The addition of SBS as a compatibilizer, partially replacing the PE or EVA fractions in the mixed systems investigated, although strongly improving the impact strength of these systems, did not generate products with as high an impact strength as that obtained with the PS/PB system. A comparison of the impact properties of the mixed compatibilized grafted systems with a similar system (same composition) obtained by mechanical blending ${ }^{18}$, shows that better impact properties are obtained with grafted systems, as expected. This is generally attributed to more efficient mixing and dispersion, as well as to the more adequate particle size (smaller and more homogenous) obtained with the latter ${ }^{15}$.

Due to limitations of equipment time, stress cracking resistance testing was conducted only for the PS/PB and compatibilized systems, i.e. those containing SBS, as these exhibited the best overall impact properties among the systems investigated. The results presented in Table 2 cle-

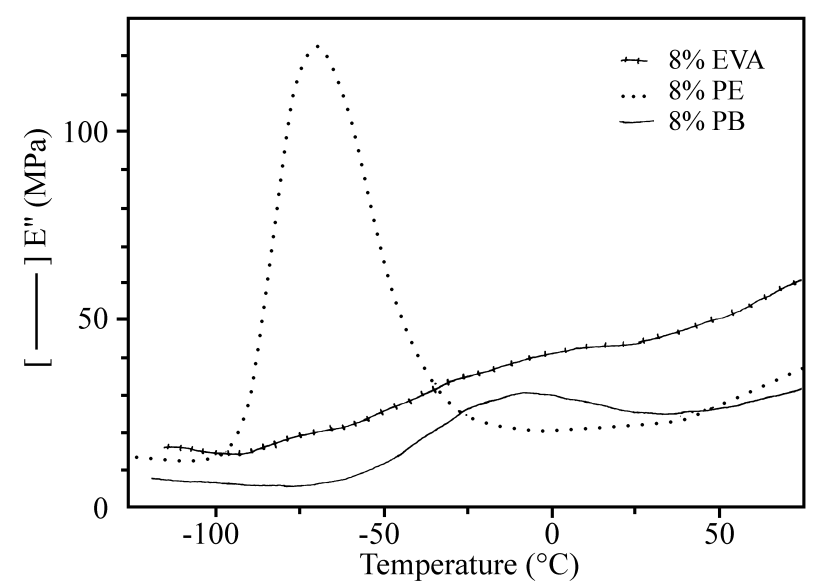

Figure 1. Curves of E" vs. temperature for PS with $8 \% \mathrm{~PB}, 8 \% \mathrm{PE}$, and $8 \%$ EVA.

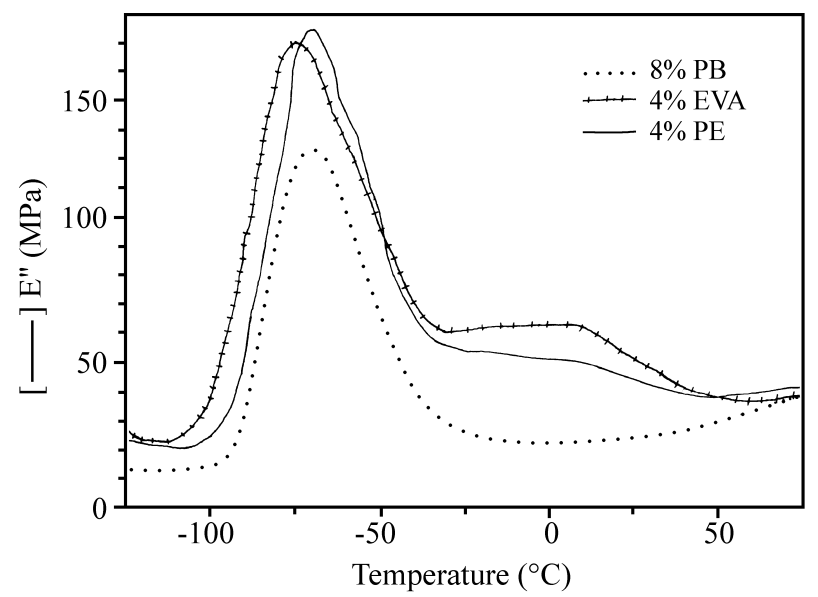

Figure 2. Curves of E" vs. temperature for PS with $8 \% \mathrm{~PB}, 4 \% \mathrm{~PB}+$ $2 \% \mathrm{PE}$, and $4 \% \mathrm{~PB}+2 \%$ EVA. arly show that small additions (2\%) of PE or EVA can improve this property two or four times its original value, respectively. This is very interesting for food packaging industries, as suitable tough materials with much greater stress-cracking resistance can thus be obtained.

Dynamic mechanical analyses (DMAs) were conducted on all systems analyzed. In every case there were two peaks, clearly indicating the presence of two phases - matrix and modifier. It can be seen that the values of Tg for PS were not changed, while those of the modifiers were greatly altered. DMA curves for the systems investigated are shown in Figs. 1-4. In general, if the Tg's are not altered, it can be said that the product is immiscible, while a single $\mathrm{Tg}$ indicates a completely miscible system. Wide peaks, in general, are due to heterogeneities within the system. Observing Figs. 1-4, it becomes evident that the sharpest peak and the one with the lowest $\mathrm{Tg}$ is that of $\mathrm{PB}$, and that the PE and EVA peaks are wider, which is attributed to the partial crystallinity of polyethylene. Figure 2 illustrates the effect of the addition of PE and EVA to the PS/PB systems. It is

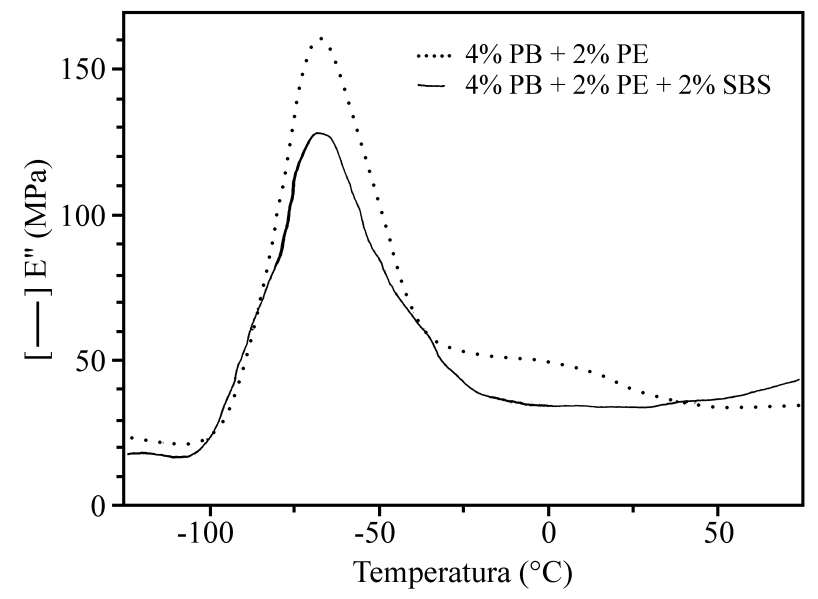

Figure 3. Curves of $\mathrm{E}^{\prime \prime} v s$. temperature for PS with $4 \% \mathrm{~PB}+2 \% \mathrm{PE}$, and $4 \% \mathrm{~PB}+2 \% \mathrm{PE}+2 \%$ SBS.

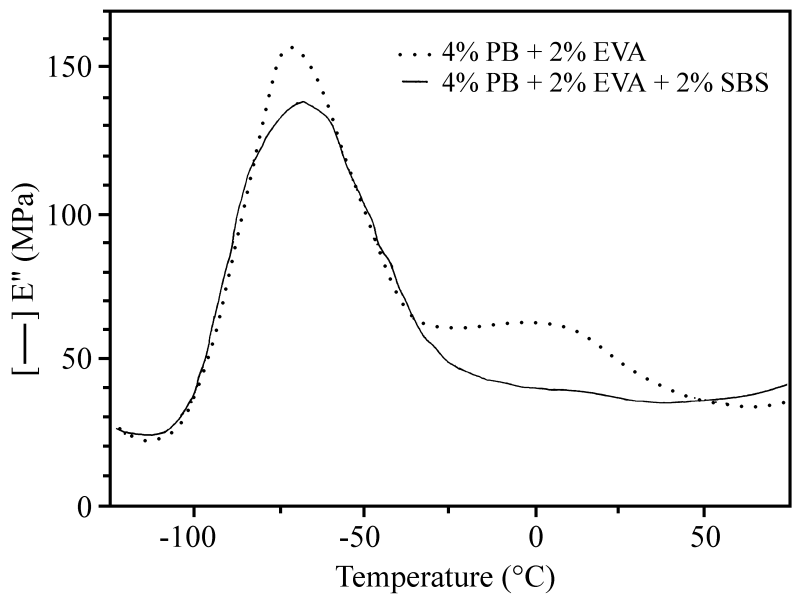

Figure 4. Curves of E" vs. temperature for PS with $4 \% \mathrm{~PB}+2 \% \mathrm{EVA}$, and $4 \% \mathrm{~PB}+2 \% \mathrm{EVA}+2 \% \mathrm{SBS}$. 
evident that the mixture of modifiers causes broadening of the PB peak, and that this effect is more pronounced for the $\mathrm{PB} / \mathrm{EVA}$ system. Broadening of the $\mathrm{PB}$ peak is attributed to compatibilization. Figures 3 and 4 show the influence of SBS addition on the mixed modifier systems. It can be seen in comparing $\mathrm{PS} / \mathrm{PB} / \mathrm{PE}$ and $\mathrm{PS} / \mathrm{PB} / \mathrm{EVA}$ systems with and without SBS that the latter ones exhibit a single Tg for the modifiers, which is taken as evidence that SBS behaves as a compatibilizing agent for the systems investigated, and that its compatibilizing action is more efficient when $\mathrm{PB}$ or EVA are used as impact modifiers.

\section{Conclusions}

PE and EVA (8\%) are not good impact modifiers for PS. However, if added in a 1:1 proportion with $\mathrm{PB}$, products with excellent chemical stress-cracking and adequate overall impact and tensile properties can be obtained. These properties can be further improved if SBS is used as a compatibilizing agent, partially substituting PE and EVA in the formulation, so that the overall content of the impact modifier remains constant (8\%). The study confirms that in comparison with mechanical blends, better impact properties are obtained with graft copolymerized systems. This is attributed to better mixing, dispersion, smaller particle size, and narrower particle size distribution obtained by synthetic routes.

\section{Acknowledgments}

The authors wish to express their gratitude to the Conselho Nacional de Desenvolvimento Científico e Tecnológico $(\mathrm{CNPq})$ and the Programa de Apoio ao Desenvolvimento Científico e Tecnológico (PADCT) for financial support, and to the Fundação Coordenação de Aperfeiçoamento de Pessoal de Nível Superior (CAPES) for a fellowship for A.M.C.R.

\section{References}

1. Rodriguez, F. In Principios de Sistemas de Polímeros; Editorial El Manual Moderno: México, 1984.
2. Grancio, M.R.; Stewart, D.F; Cass, J.F. 1983, U. S. Pat. 4386178 (for Sweetheart Plastics, Inc.).

3. Krause, S. J. Macromol. Sci., Revs. Macromol. Chem. 1972, C7(2), 251.

4. Locke, C.E.; Paul, D.R. J. Appl. Polym. Sci. 1973, 17, 2597.

5. Fayt, R.; Jerome, R; Teyssie, Ph. J. Polym. Sci., Polym. Letters 1981, 19, 79.

6. Fayt, R.; Hadjiandreou, P.; Teyssie, Ph. J. Polym. Sci., Polym. Chem. 1985, 23, 337.

7. Fayt, R.; Jerome, R.; Teyssie, Ph. J. Polym. Sci., Polym. Phys. 1982, 20, 2209.

8. Fayt, R.; Jerome, R.; Teyssie, Ph. J. Polym. Sci., Polym. Letters 1986, 24, 25.

9. Fayt, R.; Jerome, R.; Teyssie, Ph. Makromol. Chem. 1986, $187,837$.

10. Ruiz, M.E.; Bianchi, M.; Leite, P.F.; Garcez, M.P. 1989, PI 8902363 (for EDN).

11. Bucknall, C.B. "Toughened Plastics", In Applied Science; London, 1987.

12. Encyclopaedia of Polymer Science and Engineering; John Wiley \& Sons, New York, 1989; Vol. 16, p. 1.

13. Baer, M. J. Appl. Polym. Sci. 1972, 16, 1109.

14. Van Krevelen, D.W. In Properties of Polymers, Their Estimation and Correlation Chemical Structure; Elsevier, Amsterdam, 1976.

15. Traugot, T.D. In Encyclopaedia of Polymer Science and Engineering; John Wiley \& Sons, New York, 1989, Vol.16, p. 88.

16. Trotignon, J.P.; Sanschagrin, S.; Piperaud, M; Verdu, J. Mineral Fillers in Polymers, 1981, 10.

17. Trotignon, J.P. In Anais do II Congresso Brasileiro de Polímeros, São Paulo, 1991, 1.

18. Rocha, A.M.C. In Modificação de poliestireno com poliolefinas - Polietileno e Copolímeros Etileno Acetato de Vinila; Master's Thesis, Universidade Federal da Bahia, Salvador, Bahia, Brasil, 1992, 97p. 